\title{
Geoelectric structural dimensions from magnetotelluric data : Methods of estimation, old and new
}

Beamish, D., 1986. Geoelectric structural dimensions from magnetotelluric data: Methods of estimation, old and new. Geophysics, 51, 1298-1309.

doi: $10.1190 / 1.1442183$

\section{ABSTRACT}

A magnetotelluric (MT) sounding curve obtained at a given location may contain contributions from 1-D, 2-D, or 3-D geoelectric components, either singly or in combination. The modeling and interpretation of such data depend upon the ability to assess the degree of influence exerted by the three possible structural components. Six existing MT sounding curves provide case histories of the performance of both conventional and new methods for estimation of structural dimensions. For data from a single location, all the methods must be based on the horizontal rotation properties of the impedance tensor. Of the three conventional dimensional indicators considered, only one (skew) provides a degree of satisfactory performance for practical data. Three recently introduced, normalized dimensional weights appear to offer better performance. Solutions to the 1-D MT problem are central to the issue of providing dimensional constraints. The inverse theories established by Weidelt (1972) and Parker (1980) provide two tests that can be applied to establish the existence of 1-D solutions. The formalism in these theories provides the basis for a systematic method for investigating the dimensional properties of practical MT data.

\section{INTRODUCTION}

Assessment of geoelectric structural dimensions from observed magnetotelluric (MT) data is an important step in interpreting such data. The term "data" here refers to a processed MT response function of tensor impedance elements obtained as a function of frequency; such data are also referred to as an "MT sounding curve." The data may contain contributions from one-dimensional (1-D), two-dimensional (2-D), or three-dimensional (3-D) structural components, either singly or in combination. The contributions may be present only across a certain interval or they may persist over the whole frequency range. The dimensionality obviously governs the type of modeling to be applied. If several sets of data are obtained along a profile or at a high spatial density, then a further degree of control on structural dimensions can be achieved. The dimensionality analysis made possible by profile and gridded data was recently considered by Ranganayaki (1984). Here I refer to the more difficult problem of assessing dimensionality at a series of isolated points (i.e. sounding sites) which may traverse unrecognized crustal units. In fact, it is common to obtain such data before making more detailed observations.

The present study uses existing MT data obtained from six locations in southern Scotland and northern England. The site locations in relation to geology and known major crustal 
features are shown in Figure 1 . The data consist of accurate and unbiased impedance elements over the period range 30 to $7200 \mathrm{~s}$. Data processing was described in Beamish (1985a). From an initial assessment of structural dimensionality, it was concluded that a 1-D inversion of the rotationally invariant, effective response data at the three central sites ( $A, B$, and $C$ in Figure 1) was justified over the period interval 30 to $400 \mathrm{~s}$. Two 1-D inverse construction algorithms were applied. The results identified a particularly straightforward crustal profile consisting of an intracrustal, highly conducting layer dipping steeply to the northwest (Beamish, 1985a).

Assessment of structural dimensionality from MT data at a poi nt is based on the horizontal rotation proper ties of the impedance tensor. Such properties may be obtained graphically (Berdichevsky, 1968) or parametrically (Word et al, 1971). Common dimensional indicators include the skew, eccentricity, and ellipticity. To be useful, a parameter level (e.g., a departure from zero) must be defined which indicates the onset of a particular dimensional contribution.

I review estimation of such conventional parameters and their application to the six data sets. More recently introduced dimensional weights are also considered, together with estimates of the anomalous vertical field. In practice, for point data there still are substantial difficulties in providing parameters which permit unambiguous assessment of the degree of two-dimensionality or three-dimensionality. The problem is formidable and should not be underestimated. Solution requires consideration of the results of a wide variety of 2-D and 3-D modelling studies defining typical structural categories. The problem is compounded because the magnitudes of the parametric effects observed are a function of frequency and location and are therefore strongly model-dependent. Such generalities (together with the fact that certain data contributions to the parameters are inherently noisy) result in a set of parameter magnitude criteria that are necessarily ad hoc. It appears that since the role of structural dimensionality is central to correct interpretation of MT data, any further constraints are wort $\mathrm{h}$ investigation. Given the complexity of the problem, however, only marginal rather than definitive solutions are expected.

The concept of the 1-D layered geoelectric profile is the basis for interpretation of MT data. Conductivity variations with depth generate characteristic gradients and turning points within the sounding curve. When the data can be shown to be 1-D, the geoelectric profile over a given depth interval can be readily investigated. The 2-D and 3-D forward modeling studies also indicate that in certai $n$ circumstances representative 1-D profiles can be investigated and recovered from data with 2-D and 3-D characteristics.

Such 1-D models are often generated to provide the basis of initial 2-D model investigations. The response data can therefore always be viewed as a 1-D sounding curve which is perturbed to some degree by lateral variations in conducti vity structure which may be 
2-D or 3-D. From this viewpoint, such structure "distorts" the basic (1-D) sounding curve. This view motivated investigation and classification of 2-D and 3-D distortion effects undertaken at the Moscow State University and summarized in Berdichevsky and Dmitriev (1976) and Rokityansky (1982). An important class of forward models are near-surface 2$D$ and 3-D structures. Bot $h$ types of electrically $t$ hin structures can produce static (frequency-independent) distortion of a sounding curve. Such thin structures should be distinguished from substantial 2-D and 3-D structures producing frequency- dependent distortion of the equivalent 1-D sounding curve. Fortunately, it is possible to distinguish static distortion effects with an appropriate presentation of phase data.

The 1-D MT problem is central to providing dimensional constraints from MT data at a point. Even this basic problem is nonlinear and should be treated with respect. Parker (1983), in reviewing the MT inverse problem, addresses the question of the existence of 1-D solutions. I understand that a satisfactory theory is now available to determine whether or not a given finite collection of response data is consistent with any 1-D conductivity profile. If this theory can be satisfactorily applied to practical MT data, it should be important in the assessment of structural dimensionality. The theory of Parker (1980) [reviewed i n Parker (1983)] establishes a test that is necessary and sufficient for 1-D solutions to exist. The theory therefore compliments Weidelt's (1972) inequality constraints which the data and its first and second derivatives must satisfy for a 1-D solution to exist. I consider the behavior of the data in both sets of tests.

\section{DIMENSIONALITY INDICATORS}

Many authors have considered parameters relating to geo- electric structural dimensionality (Sims and Bostick, 1969; Word et al., 1971 ). Only the basic definitions are reproduced here. The MT impedance tensor ( $\underline{Z}$ ) obtained in the measured directions is given by

\section{$E=\underline{Z} . \mathbf{H}$}

with an implied dependence on frequency throughout. The electric field vector comprises the orthogonal components $\left(E_{x}, E_{y}\right)$, and $H$ is the corresponding magnetic field vector consisting of $\left(\mathrm{H}_{\mathrm{x}}, \mathrm{H}_{\mathrm{y}}\right)$. The dimensionality indicators are based on the horizontal rotation properties of $\underline{\mathbf{Z}}$. If $\underline{\mathbf{R}}$ is a coordinate rotation matrix given by

$$
\underset{\sim}{\mathbf{R}}=\left[\begin{array}{rr}
\cos \theta & \sin \theta \\
-\sin \theta & \cos \theta
\end{array}\right]
$$

for clockwise rotation through an angle $\theta$, the rotated impedance matrix (denoted by a prime) is given by 


$$
\mathbf{Z}^{\prime}(\theta)=\mathbf{R Z} \mathbf{R}^{-1}
$$

The loci of the elements of $\underline{Z}^{\prime}(\theta)$ in the complex plane are, in general, elliptical. The representation of such rotation properties forms a practical method for assessing structural dimensionality. Word et al. (1971) and Kaufman and Keller (1981) summarized the properties of several parameters generated by 1-D, 2-D and 3-D structures. The parameter combinations $\left(Z_{x x}+Z_{y y}\right)$ and $\left(Z_{x y}-Z_{y x}\right)$ are independent of $\theta$, as is their ratio. I define two rotational invariants

$$
2 Z_{1}=Z_{x y}^{\prime}(\theta)-Z_{y x}^{\prime}(\theta)=Z_{x y}-Z_{y x}
$$

And

$$
2 Z_{2}=Z_{x x}^{\prime}(\theta)+Z_{y y}^{\prime}(\theta)=Z_{x x}+Z_{y y}
$$

$Z_{1}$ and $Z_{2}$ correspond to the centers of the rotation ellipses i $n$ the complex plane. The major and mi nor axes of the $Z^{\prime}(\theta)$ ellipse are given by $M_{1}$ and $M_{2}$, respectively, wit $h$

$$
2 M_{1}=Z_{x y}^{\prime}\left(\theta_{0}\right)+Z_{y x}^{\prime}\left(\theta_{0}\right)
$$

And

$$
2 M_{2}=Z_{x x}^{\prime}\left(\theta_{0}\right)-Z_{y y}^{\prime}\left(\theta_{0}\right)
$$

where $\theta_{0}$ is the angle between a measurement coordinate axis and the direction in which $\left|M_{1}\right|$ is a maximum. $\theta_{0}$ and $\left(\theta_{0}+90\right.$ degrees $)$ are principal directions. A number of dimensional indicators have been derived based on the above parameters. The most extensively used parameter is skew $\alpha$, defined as

$\alpha=\left|Z_{2} / Z_{1}\right|$

which is zero for 1-D and 2-D structures and nonzero for general 3D structures.

As noted in Kao and Orr ( 1982) the upper limit of the value of $\alpha$ for a 3-D structure has not been clearly defined. The 3-D forward modeling studies of Reddy et al. (1977a), Ting and Hohman n (1981), and Park et al. (1983) provide upper li mits of 0.4, 0.12, and 0.5, respectively. The results of Hermance (1982) indicate that the magnitude of $\alpha$ for values between 0.001 and 0.72 is not an accurate guide to the level of near-surface distortion typically experienced by the induced electric field. Despite such difficulties, the presentation of $\alpha$ as a useful guide to dimensionality remains a common feature of many MT studies. 
The skew values for the six sites of the present study are presented on a logarithmic scale in Figure 2. The values display a typical wide range of values. It is evident that a skew value of 0.3 , shown as a horizontal line in Figure 2 , separates sites $A, B$, and $C$ with relatively low values of skew from sites I, 2 , and 3 which possess consistently higher values.

Two other indicators of 3-D structure are eccentricity and ellipticity. The eccentricity of the rotation ellipse was defined by Word et al. (1971) as

$$
\beta(\theta)=Z_{x x}^{\prime}(\theta)-Z_{y y}^{\prime}(\theta) / Z_{x y}^{\prime}(\theta)+Z_{y x}^{\prime}(\theta),
$$

which is clearly dependent upon the rotation angle. However, if $\beta(\theta)$ is evaluated at $\theta_{0}$, $\beta\left(\theta_{0}\right)=0$ is obtained for a 2-D strcture. The ellipticity $\left(\beta_{0}\right)$ defined by Word et al. (1971) as

$$
\beta_{0}=\beta\left(\theta_{0}\right)=M_{2} / M_{1}
$$

is the ratio of minor to major axes of the rotation ellipse. Nonzero values for I $\beta_{0}$ I may therefore indicate 3-D structure. The values obtained for $\left|\beta_{0}\right|$ at the six sites are shown in Figure 3. As with the val ues obtained for skew, it is necessary to establish a magnitude level which indicates the onset of 3-D behavior. The most consistent set of low values is obtained at site 2. However, from Figure 2, note that the skew values obtained at this site are among the largest from the six sites. This empirical evidence seems to support Herma nce ( 1982) who commented on his modeling results that "the utility of $\beta_{0}$ as an unequivocal indicator of three dimensionality is not apparent."

Noting the problems inherent in the interpretation of skew, Kao and Orr (1982) introduced a set of three normalized dimensional weights ( $1, D 2$, and D3 ). These weights attempt to assess the relative importance of 1-D, 2-D. and 3-D structural contributions simultaneously. With reference to the parameter definitions, the weights are defined as

$$
\begin{aligned}
& D 1=\left|Z_{1}\right| / S, \\
& D 2=\left|M_{1}\right| / S, \\
& D 3=\left|Z_{2}\right| / S,
\end{aligned}
$$

and

$$
D 3^{\prime}=\left|M_{2}\right| / S,
$$

and $\mathrm{S}$ is defined as

$S=\left|Z_{1}\right|+\left|M_{1}\right|+\left(\left|Z_{2}\right|+\left|M_{2}\right|\right) / 2$. 
The dimensional weights D1, D2, and D3 for the six sites are shown in Figure 4.

Dimensional weight D3' is not considered. It is evident that for sites $A, B$, and $C$ the condition D1 » D2 > D3 is generally true. The weights at the three remaining sites display very different behavior. The largest dimensional weight at sites 1 and 2 is D2. The reciprocal behavior of the D1 and D3 weights with period at site 2 is a location characteristic. The behavior of the three weights is consistent with the dimensional characteristics infer red from the behavior of skew at the six locations. The dimensional weights, however, appear to offer a direct, simultaneous assessment of the relative contributions from 1-D, 2-D, and 3-D structural components.

For measurements which include the vertical field, the vertical field transfer function, or tipper, may provide further dimensional constraints. In a true 1-D situation, the vertical field transfer function is zero. When the vertical field transfer function is nonzero, its horizontal rotation properties define a relatively unambiguous geoelectric strike direction $\left(\theta_{S}\right)$. In 2-D situations, determination of $\theta_{S}$, removes the ambiguity of 90 degrees inherent in determination of geoelectric strike using the MT principal directions. It is also evident that in strongly 3-D situations, the geoelectric strike determined by the two methods may differ by a substantial angle (Jones and Vozoff. 1978). The magnitudes of the vertical field transfer function at sites $A$. B, and $C$ with a large D1 weight and at site 1 with a large D2 weight are shown in Figure 5. The consistent behavior of the vertical field at sites $A, B$, and $C$ for periods greater than $400 \mathrm{~s}$ is a regional induction effect (Banks and Beamish, 1984). At periods less than $400 \mathrm{~s}$, the vertical field magnitude at sites $A, B$, and $C$ is small (i.e., < 0.15 ), while the strong frequency dependence and large magnitude at site 1 is thought to reflect strong 2-D behavior (Beamish, 1985b, Figure 10c). A comparison of the azimuths $\theta_{0},\left(\theta_{0}+90\right.$ degrees), and $S$, at the six sites reveals little of the behavior predicted by 2-D models; so azimuth assessment appears to be the least effective way to assess structural dimensionality.

\section{1-D, 2-D, AND 3-D FORWARD MODELS}

Given an MT response function with either 2-D or 3-D characteristics, it is still considered acceptable, in some cases, to establish 1 -D models of geoelectric structure provided the results of appropriate forward models are considered. Any discussion of the magnitude of 2-D and 3-D effects is inevitably a complicated function of the dimensions of the structure, the point of observation, and t e frequency interval considered. It is still possible, however, to establish certain general characteristics particularly regarding the phase.

A strictly 2-D earth with given horizontal strike provides two orthogonal, uncoupled response modes referred to as TE (electric field parallel to strike) and TM (magnetic field parallel to strike). The characteristic feature of the response is displacement of the 
magnitude the response determined in the two principal directions. The 2-D modeling studies by Madden and Swift (1969) and Wright (1970) showed that the sounding curve determined along the TE direction is more representative of an equivalent plane-layered 1-D model. As a general result of such relatively simple 2-D models (Reddy and Rank i $n$, 1972: Reddy et al., 1977b), the major (maximum) principal direction will be parallel to strike on the conductive side of the geoelectric contrast and perpendicular to strike on the resistive side of the contrast. In this strictly 2-D situation, the problem essentially reduces to determination of geoelectric strike direction. In addition to displacement of the magnitude of the response in the two principal directions, the modeling studies also reveal that across the region where this displacement is most pronounced, a frequency dependence of both TE and TM mode phase data is generally observed which is different for the $t$ wo modes. The region across which these effects are most pronounced is also associated with the largest vertical fields.

Near-surface 2-D and 3-D models were considered by Berdichevsky and Dmitriev (1976), Hermance (1982), and Park et al. (1983), among others. These studies introduced an electrically thin, laterally inhomogeneous surface layer with either 2-D or 3-D characteristics and the effects of that layer on equivalent 1-D and 2-D MT sounding curves. The models essentially consider the problem of static distortion (Hermance, 1982). Near-surface conductivity variations of limited extent typically cause vertical displacement of both TE and TM resistivity curves, but the phase data are unaffected. The most representative 1-D curve in such circumstances is close to, but below, the major resistivity curve, both in the TE mode direction and in the TM mode direction (Berdichevsky and Dmitriev, 1976). In the absence of additional constraints, Berdichevsk y and Dmitriev (1976) demonstrated that a useful procedure is to form an average or effective impedance constructed from the linear average of the impedances in the two principal directions as

$$
Z_{E}=\left(Z_{x y}-Z_{y x}\right) / 2,
$$

which is rotationally invariant. The procedure provides an effective resistivity curve biased toward the maximum of the bilaterally displaced sounding curves.

The above discussion of the results of forward models suggests that if the dimensional characteristics of the tensor response data can be established, there are appropriate procedures for recovery of equivalent 1-D profiles. If the data exhibit strong 2-D characteristics, the response in a direction parallel to geoelectric strike should be used. If the data exhibit strong 3-D characteristics, the rotationally invariant effective response should be employed. The difficulties i n providing parameters which permit a quantitative assessment of the degree of 2-D and 3-D contributions have already been noted. The above discussion also suggests, however, that the phase data for the TE and TM modes may provide a level of arbitration in identification of dimensional contributions. In practice, the rotation of MT tensor data in to principal directions will usually provide major and minor response curves 
although in a 1-D situation the two curves may be numerically identical. The major and minor response data (resistivi ty and phase) for the six sites are shown in Figure 6. Both 2-D and 3-D structural contributions can produce vertical displacement of the two curves. If only near-surface 3-D contributions are present, the two resistivity curves will be displaced parallel and the phase data will be identical and representative of the equivalent 1-D profile. The response data at sites $A, B$, and $C$ have already been characterized as having low values of skew, large D1 weights, and small vertical fields. For these three data sets the major and minor resistivity curves exhibit a degree of parallelism i $n$ the lower period interval. At periods greater than $1000 \mathrm{~s}$, the two curves diverge at all three sites. To conform with the model, the major and minor phase curves should be identical. The phase data from all six sites depart from the ideal; however, the degree of departure appears least at sites A, B, and $C$ for periods less than $400 \mathrm{~s}$. I suggest that these characteristics can be usefully interpreted as at least partially due to near-surface 3-D effects operating in the period range 30 to $400 \mathrm{~s}$. At periods greater than $1000 \mathrm{~s}$, the interpretation is less clear. The data at sites 1,2 , and 3 have already been shown to exhibit large values of skew and a variety of levels in the three dimensional weights (DI, D2, and D3). The response at site 1 is associated with a substantial vertical field. It is fairly evident from Figure 6 that no simple interpretation is possible at these three sites. It could be suggested that the characteristics observed at site 1 in Figure 6 may be due to a combination of near-surface 3-D and substantial 2-D contributions. At present there is no way of separating such complex contributions from response data at a point.

The dimensional indicators presented for sites 1, 2, and 3 are complex enough that direct recovery of 1-D profiles from the data does not appear warranted, with the possible exception of data from site 1 which appear to possess a strong 2-D component. For further analysis, the dimensional characteristics at the three sites $A, B$, and $C$ over the period 30 to $400 \mathrm{~s}$ are considered representative of a strong 1-D contribution distorted by near-surface 3-D structural contributions. Accordingly, the effective impedance over this period is now used as a basis for further assessments of structural dimensionality.

The 1-D electromagnetic (EM) induction problem requires consideration of the behavior of the electric field induced in material of finite conductivity $\sigma=\sigma(z)$ by a periodic and uniform source field varying as $e^{i \omega t}$. Within a 1-D earth, the complex electric field $\mathbf{E}$ obeys the differential equation

$$
d^{2} \mathbf{E}(z, \omega) / d z^{2}-i \omega \mu_{0} \sigma(z) \mathbf{E}(z, \omega)=0
$$

If $\sigma(z)$ is known, the complex response function $c(\omega)$ can be calculated as

$$
c(\omega)=-\mathbf{E}(z, \omega) / \mathbf{E}^{\prime}(z, \omega)
$$


observed at the earth's surface $(z=0)$. The complex response is readily transformed into the more familiar $M T$ impedance $\mathbf{Z}$ as

$$
c(\omega)=g(\omega)-i h(\omega)=Z(\omega) / i \omega \mu_{0} .
$$

The 1-D inverse problem considers what can be deduced about $\sigma(z)$ given a finite set of observations of $c(\omega)$. The first question raised by this problem is: Does there exist any 1-D distribution of conductivity which will reproduce the observations?

\section{INEQUALITY CONSTRAINTS}

Analytical properties of the complex response e(co) are described by the general theory of second-order, linear differential equations. These equations were considered in detail by Weidelt (1972) who established the general relationship

$$
c(\omega)=a(\lambda) d \lambda /(\lambda+i \omega)
$$

where $\mathrm{a}$ is a bounded nondecreasing real function. This transformation allowed Weidelt to deduce all of the analytic properties obeyed by the complex response function. Many of the properties have since been discussed in Rokityansky (1982) and Parker (1983). The simplest property of the relationship $c(\omega)=g(\omega)$ - ih $(\omega)$ is that the real and imaginary parts must be nonnegative. Using a mechanical analogy, the positive length $g(\omega)$ can be interpreted as the depth of the center of gravity of the in-phase induced current system.

By introducing the differential operator $D$ defined as

$$
D f=\omega d f / d \omega=d f / d(\log \omega),
$$

Weidelt established 19 inequality constraints which must be upheld by 1-D data. The first nine inequality constraints (I1 to I9) are

$$
\begin{array}{lr}
g \geq 0, \quad h \geq 0, & (I 1, I 2) \\
D g \leq 0, & (I 3) \\
0 \leq-D|c| \leq|c|, & (I 4, I 5) \\
|D c| \leq h, \quad|c+D c| \leq g, & (I 6, I 7) \\
\left|D^{2} c\right| \leq h, \quad \text { and }\left|c+2 D c+D^{2} c\right| \leq g . & (I 8, I 9)
\end{array}
$$

Each of these conditions must be upheld at every frequency. Clearly constraints I3 to 19 involve the first and second derivatives of the observed complex response function. 
Although the derivatives cannot be determined using only a finite number of observations, they can nevertheless be estimated. The derivatives can typically be obtained using the derivative of a Lagrangian interpolation polynomial (Jones, 1980). However, note that such a numerical procedure is inherently unstable The approximate values of the derivatives obtained will, in general, be less accurate than the functions from which they are obtained.

Two main problems are associated with the inequality constraints given above. The first problem is that the data have errors that must ultimately be taken into account in order to provide valid assessments concerning existence of solutions. The second problem is that other functions, which are not admissible as valid 1-D response functions, may also satisfy the inequality constraints (Parker, 1983).

The required quantities were obtained from the response functions at all six sites using a three-point formula operating on the effective impedance over the period interval from 30 to $400 \mathrm{~s}$. The performance of the inequality constraints on the data from site B is illustrated in Table 1. Of the $9 \times 8=72$ inequalities tested, 83 percent were found to be accurate. The corresponding performance results at the six sites are: 82 per-cent (site A), 83 percent (B), 90 percent (C), 93 percent (1), 53 percent (2), and 19 percent (3). Given the instability of the numerical procedure, it is difficult to attach a level of significance to such pass marks. In certain circumstances a period range over which a 1-D response would not be appropriate might be established. However, this does not arise for the present data. Clearly a 1-D interpretation of the response data at sites 2 and 3 does not appear warranted, in accord with previous dimensional indicators. Also note that site 1 , with a large D2 dimensional weight, provides the highest pass mark in this necessary test for one-dimensionality. Furthermore, note that the above inequality constraints are invariant with respect to the near-surface static distortion effects considered previously (Larsen, 1981).

\section{ONE-DIMENSIONAL INVERSION}

Parker (1983), in reviewing the 1-D MT inverse problem, addressed several relevant questions including methods for construction of solutions. Since the inverse problem is nonlinear, Parker ( 1983) noted that methods based on linearization of the problem cannot provide wholly systematic inferences concerning real Earth structure. Such difficulties can be overcome using the fully nonlinear solutions of Parker (1980) and Parker and Whaler (1981). The three classes of solutions, referred to as $\mathrm{D}+, \mathrm{H}+$, and $\mathrm{C2}+$, acknowledge the fact that the data have errors and the acceptability of any particular model is measured by the chi-square $\left(\chi^{2}\right)$ misfit. The problem of existence of a 1-D solution is reduced to finding a model with the smallest possible misfit ; if this optimum model is rejected, so will every other model capable of reproducing the data to some degree be rejected. The nonlinear theory presented in Parker (1980) establishes the fact that the optimum ( best- fit) model is a delta-function ( $D+$ ) model, consisting of a finite number of 
delta functions (zero thickness but finite conductance) separated by perfect insulators. The $D+$ solutions at the six sites for the effective impedance data are shown in Table 2 . The data consist of eight complex results over the period 30 to $400 \mathrm{~s}$, the expectation level of is $\chi^{2} 16$, and a $\chi^{2}<27$ is considered acceptable at the 95 percent confidence limit. This level is achieved by the $D+$ solutions at five of the six sites. With the exception of the solution at site 1 , all solutions terminate with a perfect conductor. Several types of behavior can be observed. At sites A, B, and C a single crustal delta function is required. The effective depth of penetration of the data was determined using the method established in Parker (1983). The results reveal that structure below $115 \mathrm{~km}$ is nonphysical. At site $\mathrm{C}$ a second, deeper delta function has been introduced to achieve a low level of misfit. The acceptable solutions obtained at sites 1 and 2 appear rather exotic.

At site 1 , the stack of delta functions provides an extremely low value of misfit. To emphasize the quality of the D+ solution obtained, the observed and calculated complex response functions are compared in Figure 7 . The data from this site provide skew values in the range 0.56 to 0.78 , a large D2 dimensional weight, and the vertical field transfer function is one of the largest to be observed in mainland (non- coastal) Britain ( Beamish, 1985b). Thus the data set cannot be compatible with a 1-D assumption. The D+ result shown in Figure 7 and in Table 2 demonstrates that more than adequate 1-D solutions can be generated from data which would not otherwise be classified as 1-D. Acceptable 1-D solutions are not limited to the effective impedance (to be discussed). In view of the results presented, it seems necessary to emphasize that the above techniques only reject the existence of 1-D solutions. It is obviously possible to construct specific 2-D and 3-D models which generate satisfactory 1-D solutions; however, a formalism which encompasses the existence or nonexistence of 1-D solutions in the case of general 2-D and 3-D structural components remains to be explored.

Thus far I have limited my study to D+ solutions derived from effective or averaged impedance data. I now consider to what extent the methods described concerning the existence of 1-D solutions can be used to infer dimensionality. Refer back to the horizontal rotation properties of general tensor impedance data described previously. For a 1-D structure the rotational properties are invariant, and clearly 1-D solutions must exist at every azimut $h$. For a 2-D structure, the rotated tensor $\underline{Z}^{\prime}(\theta)$ must diagonalize for 90 degree increments of $\theta$.

Two-dimensional structures therefore provide symmetrical properties in increments of 90 degrees. The procedure adopted was to apply the $D+$ algorithm to directional response data obtained in equal increments of 10 degrees over the interval from 0 to 180 degrees. The variation in D+ misfit as a function of rotation angle at the three 1-D data sets $(A, B$, and C) is shown in Figure 8a. The variation is sufficiently small that a linear scale can be used; the 95 percent and 99 percent $\chi^{2}$ confidence limits are indicated by horizontal broken 
lines. The minimum $\chi^{2}$ obtained for these data are all significantly larger than those obtained using the invariant response data. Clearly, even at the 99 percent confidence limit acceptable 1-D models are not obtained at every azimuth. The results, however, establish several useful facts.

The results of Figure 8a reveal that if the data were rotated to a common azimuth (e.g., a geologically plausible azimuth such as 75 degrees east), acceptable 1-D solutions would not exist at all three sites. In fact for these data no single azimuth can be found for which acceptable solutions exist at the 95 percent limit at all three sites. Another interesting fact emerges when the principal directions $\left(\theta_{0}\right)$ at the three sites are considered in relation to the results obtained. The ranges of values of $\theta_{0}$ at each of the three sites over the same bandwidth are shown in Figure $8 a$ by the shaded regions against the corresponding rotation curves. The directional response at $\theta_{0}$ consists of the rotated major response. If a 1-D inversion of these data were attempted, Figure 8a would establish that an acceptable 1-D solution would only be obtained at one site (A). Acceptable 1-D solutions exist for the other two sites at azimuths of $\theta_{0}+90$ degrees, corresponding to rotated minor response data. Since such behavior can be systematically monitored, the method is a powerful means of investigating the dimensional properties of MT data.

An identical procedure was performed on the data at the sites 1, 2, and 3 . The equivalent results are shown in Figure $8 \mathrm{~b}$. For these results it is necessary to use a logarithmic scale for the misfit. The 95 percent and 99 percent acceptance levels, together with the azimuthal ranges of $\theta_{0}$, are again shown. The results obtained have very different characteristics from those of Figure 8a. Only at one site (1) do acceptable 1-D solutions exist over a substantial azimuthal interval. An acceptable 1-D solution occurs at an azimuth corresponding to the rotated major response data at this site. The large variation in misfit levels for these data suggests a strong departure from one-dimensionality, as has already been indicated in the various dimensional indicators presented. The symmetric rotation characteristics at sites 1 and 2 appear to imply a degree of 2-D behavior, which would appear to comply with the values obtained for the dimensional weights at these sites i.e., D2 > D1. The slightly asymmetric rotation characteristics observed at site 3 may also comply with the observation D3 $\approx$ D1.

\section{DISCUSSION}

This stud y used six widely separated data sets to investigate a number of conventional and newly established methods for assessing structural dimensionality. For the three conventional indicators considered, it has been demonstrated that skew values, although useful, do not provide an unambiguous assessment of the degree of 3-D distortion. For the present data, the low values of skew together with the low values obtained for the magnitude of the vertical field at three sites $(A, B$, and $C)$ can be used to infer 1-D 
characteristics. Note, however, that vertical field estimates are not always available. A comparison of the three normalized weights introduced by Kao and Orr (1982) appears to confirm the above inference. I recommend that studies of these weights in relation to the results of general 2-D and 3-D forward models be undertaken since the weights appear to offer a direct method for simultaneous assessment of the relative contributions from 1-D, 2-D, and 3-D structural components. Considering typical data bandwidths, however, it remains questionable whether such forward models can successfully reproduce structural contributions with dimensions ranging from hundreds of meters (e.g., static distortion) to hundreds of kilometers.

Consideration of the results of 2-D and 3-D forward models suggests that an appropriate choice of response data for the recovery of equivalent 1-D profiles can be made. The choice between rotational data at a given azimuth and rotationally invariant data depends upon whether the data exhibit 2-D or 3-D characteristics. I have also emphasized that nearsurface, static distortion effects can be identified, to a certain extent, using the phase of the sounding curves in the principal directions.

It is evident from the 1-D inverse problem that methods which consider the existence of 1-D solutions play an important role in assessment of structural dimensionality. Again such assessments must be based on the horizontal rotation properties of the impedance tensor. Because such tests must be applied to data rotated through successive azimuths, existence tests are required which are numerically robust when the data are subject to errors. The inequality constraints of Weidelt (1972) provide necessary conditions for the existence of 1D solutions. Inequality constraints were illustrated using the effective impedance data at the six sites. Their numerical implementation is straightforward but subject to error propagation ; therefore it is difficult to attach significance to the results obtained. The nonlinear theory presented in Parker (1980) reduces the problem of existence of 1-D solutions to an assessment of the acceptability of the optimum (D+ ) solution. Despite the fact that the condition requires careful, numerical implementation (Parker and Whaler, 1981), it has been demonstrated that the technique is the basis for a systematic scheme for assessing structural dimensions.

For the present data, neither the dimensional indicators nor the existence tests can provide unequivocal results regarding structural dimensions. I do not anticipate definitive answers for the reasons already outlined. It appears that point data must undergo at least some of the tests considered. The six data sets provide examples of the behavior associated with 1-D, 2-D, and 3-D structural contributions. The accumulated evidence presented for the three central sites $(A, B$, and $C)$ points to data which are strongly 1-D but which nevertheless are perturbed by the near-surface effects over the period from 30 to $400 \mathrm{~s}$. The extent to which satisfactory inferences may be made regarding real Earth structure using these data was considered by Beamish and Smythe (1986). 


\section{ACKNOWLEDGMENTS}

$M y$ thanks go to Bob Parker of the University of California for providing the original coding of his algorithms; the pro- grams have been revised to work on small-core minicom pu ter systems. This paper is published by permission of the Director, British Geological Survey (NERC).

\section{REFERENCES}

Banks, R. J. and Beamish. D., 1984, Local and regional induction in the British Isles: Geophys. J. Roy. Astr. Soc., 79, 539-553.

Beamish, D., 1985a, Deep crustal geoelectric structure beneath the Northumberland basin: Geophys. J. Roy. Astr. Soc., 84, 619-640.

1985b, The frequency characteristics of anomalous vertical fields observed in the British Isles: J. Geophys., 57, 207-216.

Beamish, D., and Smythe, D. K., 1986, Geophysical images of the deep crust: the lapetus suture: J. Geol. Soc. London, in press.

Berdichevsky, M. N., 1968, Electrical prospecting with the method of magnetotelluric profiling: Nedra, (in Russian).

Berdichevsky, M. N., and Dmitriev, V. I., 1976, Basic principles of interpretation of magnetotelluric sounding curves, in Adam, A., Ed., Geoelectric and geothermal studies: K APG Geophys. Mono., Akad. Kiado, 165-221.

Hermance, J. F., 1982, The asymptotic response of three-dimensional basin offsets to magnetotelluric fields at long periods: The effects of current channeling: Geophysics, 47, 1562-1573.

Jones, A. G., 1980, Geomagnetic induction studies in Scandinavia : I. Determination of the inductive response function from the magnetometer array data : J. Geophys., 48, 181-194.

Jones, F. W. and Vozoff, K., 1978, The calculation of magnetotelluric quantities for threedimensional conductivity inhomogeneities: Geophysics, 43, 1167-1175.

Kao, D., and Orr, D., 1982, Magnetotelluric studies in the Market Weighton area of eastern England: Geophys. J. Roy. Astr. Soc., 70, 323-337.

Kaufman, A. A., and Keller, G. V., 1981, The magnetotelluric sounding method: Methods in geochemistry and geophysics, 15: Elsevier Science Pub. Co. 
Larsen. J. C., 1981, A new technique for layered earth magnetotelluric inversion: Geophysics, $46,1247-1257$.

Madden, T. R., and Swift. C. M., J r., 1969, Magnetotelluric studies of the electrical conductivity structure of the crust and upper mantle, in Hart, P. J., Ed., The Earth's crust and upper mantle: Am. Geophys. Union, Geophysics Monograph 13, 469--479.

Park. S. K., Orange, A. S.. and Madden, T. R., 1983. Effects of three- dimensional stucture on magnetotelluric sounding curves: Geophysics. 48, 1402-1405.

Parker. R. L., 1980, The inverse problem of electromagnetic induction : Existence and construction of solutions based on incomplete data : J. Geophys. Res., 85, 4421--4428.

- $\quad$-- 1982, The existence of a region inaccessible to magnetotelluric sounding: Geophys. J. Roy. Astr. Soc.. 68. 165-170.

-- 1983. The magnetotelluric inverse problem : Geophys. Surv., 6, 525.

Parker, R. L., and W haler, K. A., 1981. Numerical methods for establishing solutions to the inverse problem of electromagnetic induction : J. Grophys. Res., 86. 9574-9584.

Ranganayaki, R. P.. 1984. An interpretive analysis of magnetotelluric da ta: Geophysics, 49, 1 730-1 748.

Reddy, I. K., and Rankin, D., 1972, On the interpretation of magnetotelluric data i $n$ the plains of Alberta: Can. J. Eart h Sci.. 9, 514-527.

Reddy, I. K., Rankin, D., and Philli ps, R. J., 1977a. Three-dimensional modelling i n magnetotelluric and magnetic variational sounding: Geophys. J. Roy. Ast r. Sot:., 51, 313325.

-- 1977b, Electrical structure in a region of the Transverse Ranges, southern California: Earth Pla net. Sci. Lett., 34, 313-320.

Rokityansky, I. I.. 1982, Geoelectromagnetic investigation of the Earth's crust and mantle: Springer-Verlag.

Sims, W. E., and Bostick, F. X., Jr., 1969, Methods of magnetotelluric analysis: EG R L Tech. Rep.. 58, Univ. of Texas Austin.

Ting, S. C.. and Hohmann, G. W., 1981, Integral equation modeling of three-dimensional magnetotelluric response: Geophysics, 46, 182-197.

Weidelt, P., 1972, The inverse problem of geomagnetic induction: Z. Geophys., 38. 257289. 
Word. D. R., Smith, H. W.. and Bostick, F. X., J r., 1971, Crustal i n vestigations by the magnetotel! u ric tensor impedance met hod, in Heacock. J. G.. Ed .. The st ruct u re and physical properties of the Eart h's crust : Am. Geophys. Union Geophysics Monograph 14, 145-167.

Wright, J. A., 1970, Anisot ropic apparent resistivities arising from non-homogenous t wodimensional st ruct u res: Can. J. Earth Sci., 7, 527-531. 
Table 1 Results obtained by applying the first nine inequality constraints (ICl to IC9) of Weidelt (1972) to eight estimates of the effective impedance for data from site B. Central periods are given in seconds. A plus sign indicates that a particular inequality constraint is satisfied.

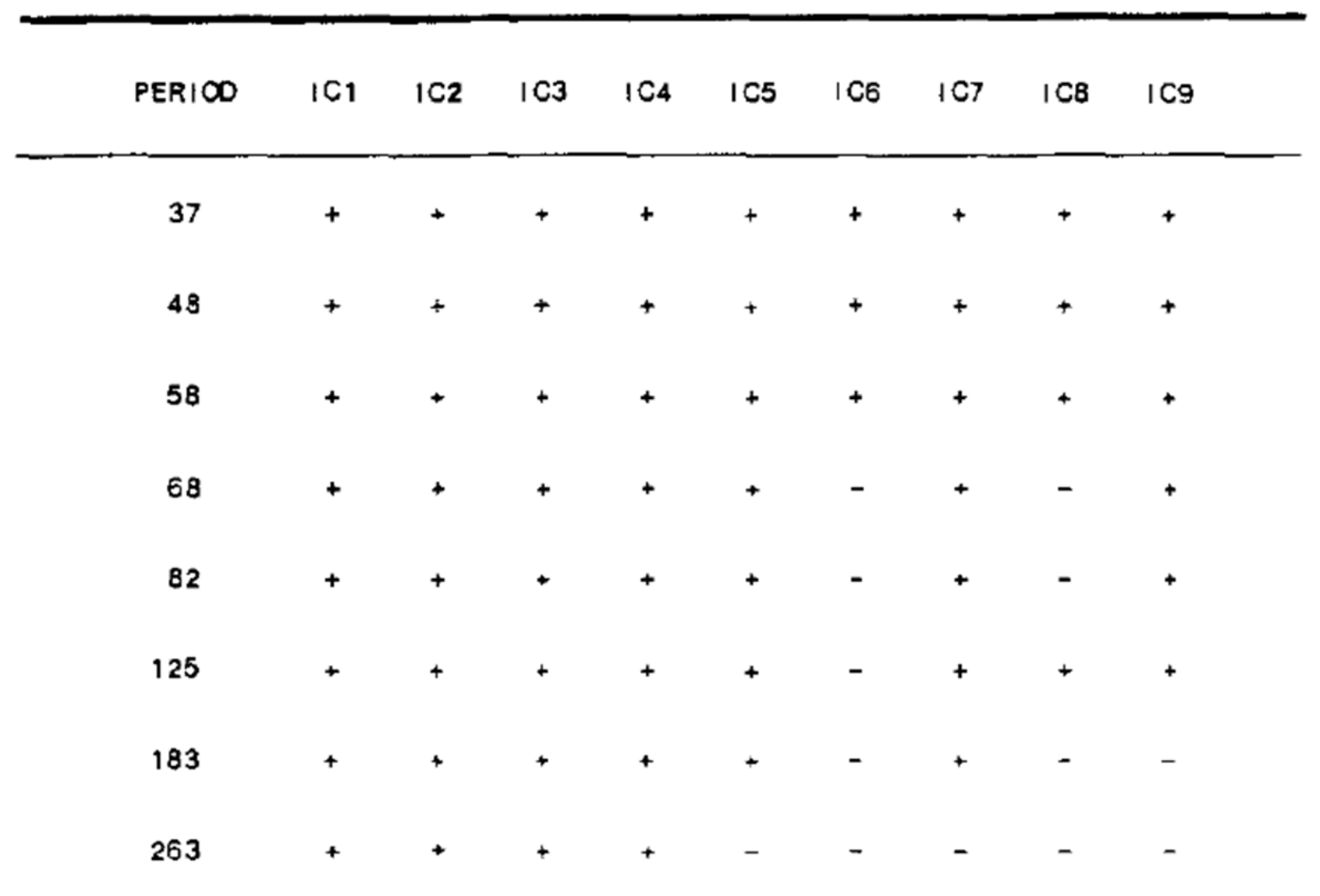


Table 2 Optimum $D+$ solutions for the effective impedance data from the six sites $A, 8, C, 1,2$, and 3. Data bandwidth is from $\mathbf{3 0}$ to $\mathbf{4 0 0} \mathrm{s}$. Delta functions obtained at a particular depth are denoted by a delta number and provide a conductance (conductivity times thickness) value. Solutions at all but site 1 are underlain by a perfect conductor at the final depth indicated.

\begin{tabular}{|c|c|c|c|c|}
\hline $\begin{array}{l}\text { Sit: } \\
\text { code }\end{array}$ & $\begin{array}{c}\text { Deita } \\
\text { no. }\end{array}$ & $\begin{array}{l}\text { Depth } \\
(\mathrm{km})\end{array}$ & $\begin{array}{l}\text { Conductance } \\
\text { (Siemens) }\end{array}$ & $\begin{array}{c}\text { ChI-square } \\
\text { mistit }\end{array}$ \\
\hline$A$ & 1 & $\begin{array}{r}29.1 \\
148.2\end{array}$ & 648.9 & 10.96 \\
\hline B & $\begin{array}{l}1 \\
2\end{array}$ & $\begin{array}{r}18.4 \\
115.9 \\
181.8\end{array}$ & $\begin{array}{l}774.1 \\
835.9\end{array}$ & 12.69 \\
\hline C & $\begin{array}{l}1 \\
2\end{array}$ & $\begin{array}{r}10.7 \\
62.9 \\
160.4\end{array}$ & $\begin{array}{l}858.2 \\
511.4\end{array}$ & 9.64 \\
\hline 1 & $\begin{array}{l}1 \\
2 \\
3 \\
4\end{array}$ & $\begin{array}{r}0.0 \\
11.6 \\
46.2 \\
77.4\end{array}$ & $\begin{array}{r}123.9 \\
548.5 \\
690.9 \\
3340.9\end{array}$ & 1.38 \\
\hline 2 & 1 & $\begin{array}{l}0.0 \\
4.4\end{array}$ & 1751.6 & 20.99 \\
\hline 3 & 1 & $\begin{array}{r}0.0 \\
77.4\end{array}$ & 1627.6 & 2429.02 \\
\hline
\end{tabular}




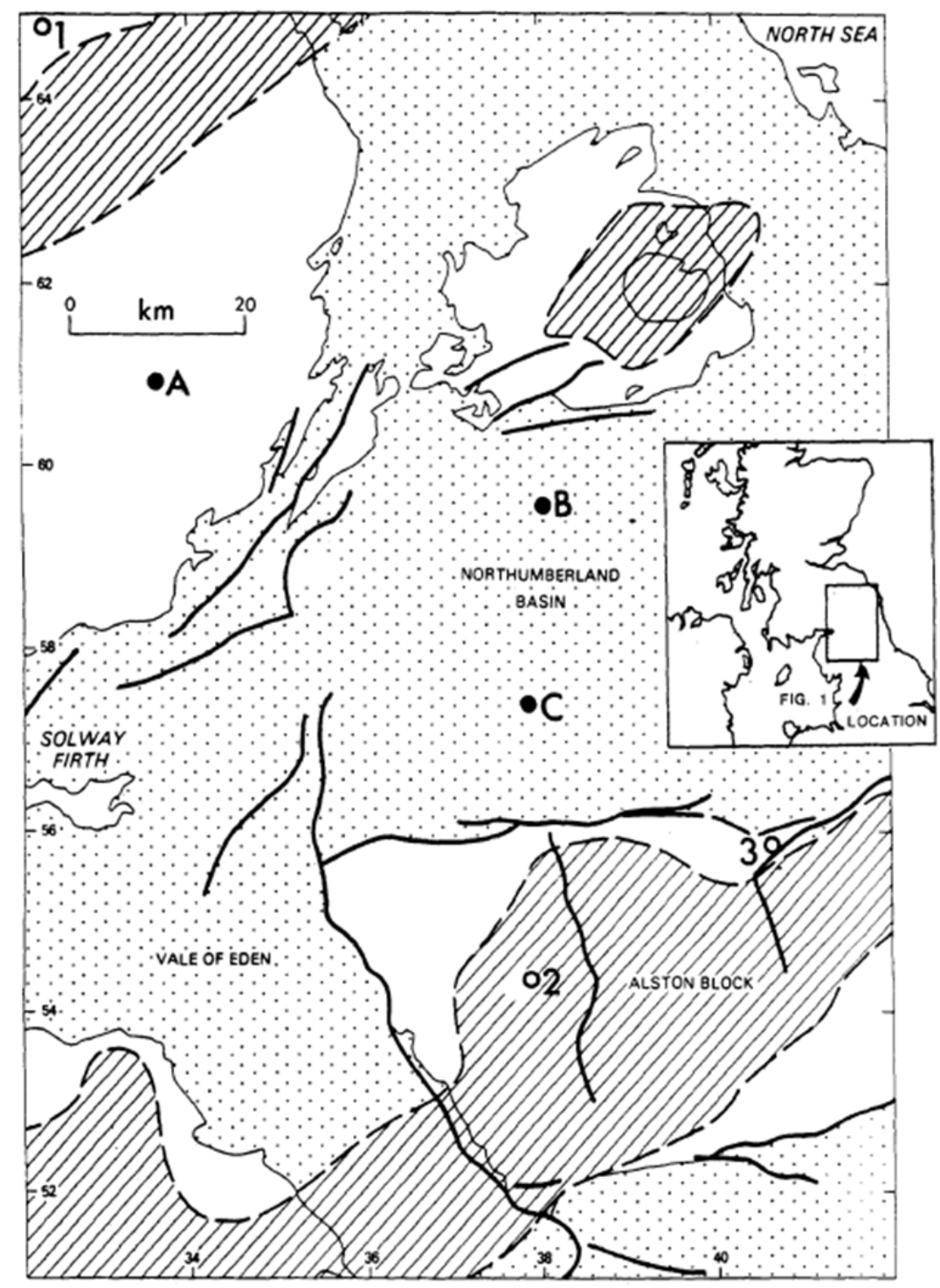

FIG. 1. Six MT sites and major geologic features of southern Scotland and northern England. Central three sites (A, B, and C) are solid circles and remaining three sites (I, 2, and 3 ) are open circles. Major faults are shown as bold lines. Shading indicates areas underlain by granite batholiths. Stippled areas are underlain by substantial thicknesses of postCaledoniansedimentary rock. Coordinate values are National Grid; multiply by 10 for kilometer scale. 


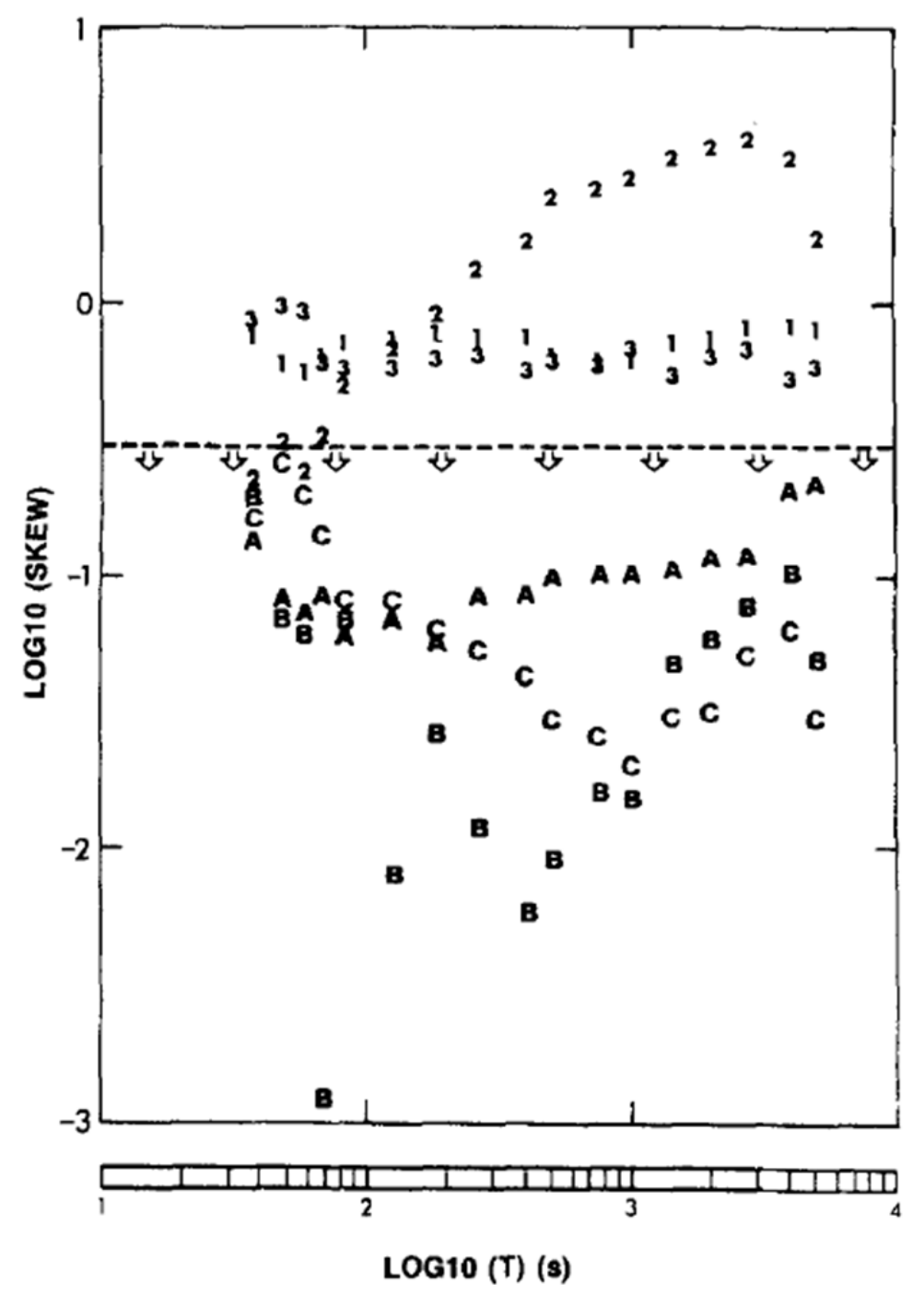

FIG. 2. Skew values for the six data sets ( $A, B, C, I, 2$, and 3 ) displayed on a logarithmic scale. Skew values are identified by the site codes. Broken horizontal line denotes a skew value of 0.3 . 

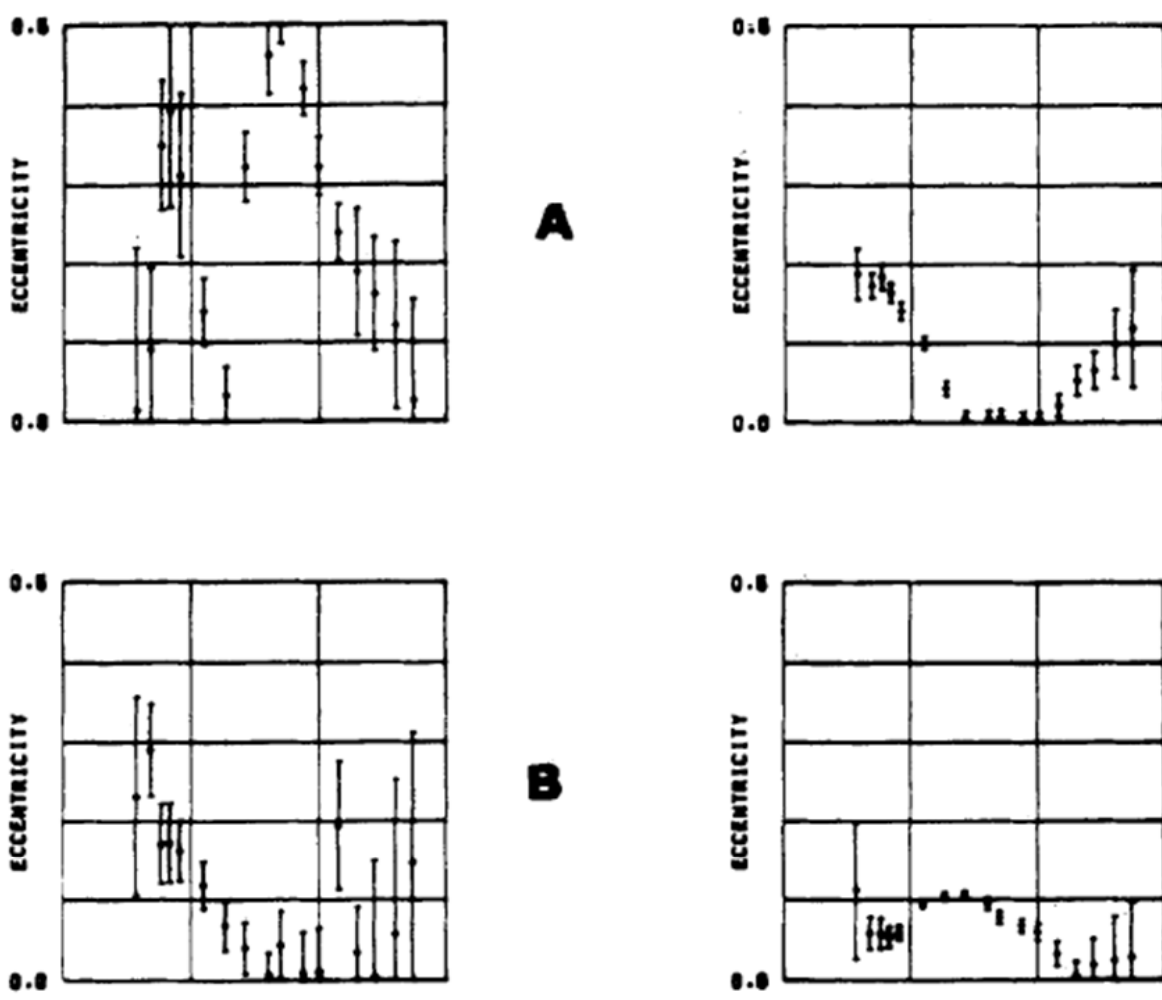

$\mathbf{B}$

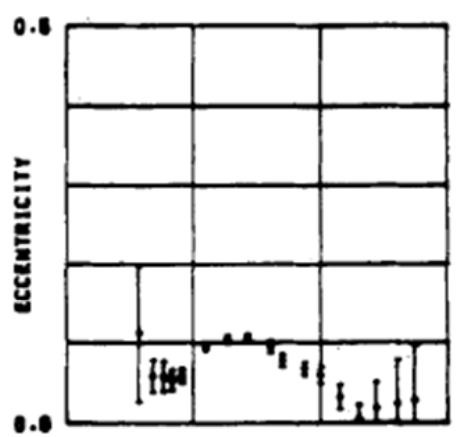

2
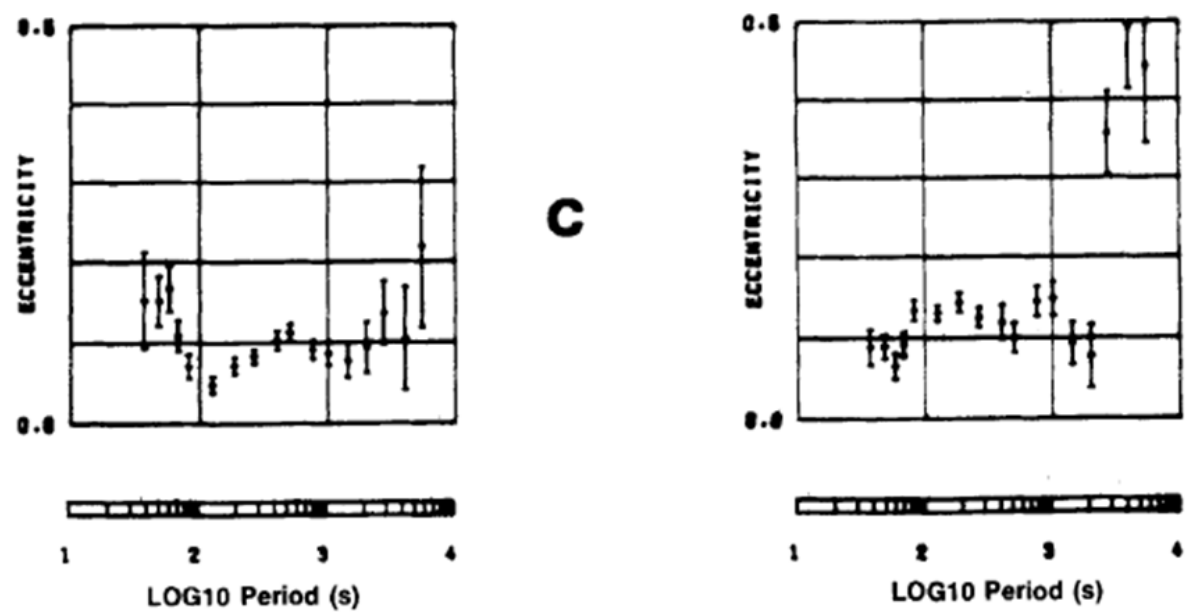

FIG. 3. Values of eccentricity evaluated in the principal direction (i.e., ellipticity) for the six data sets. Error bars refer to 68 percent confidence limits. 

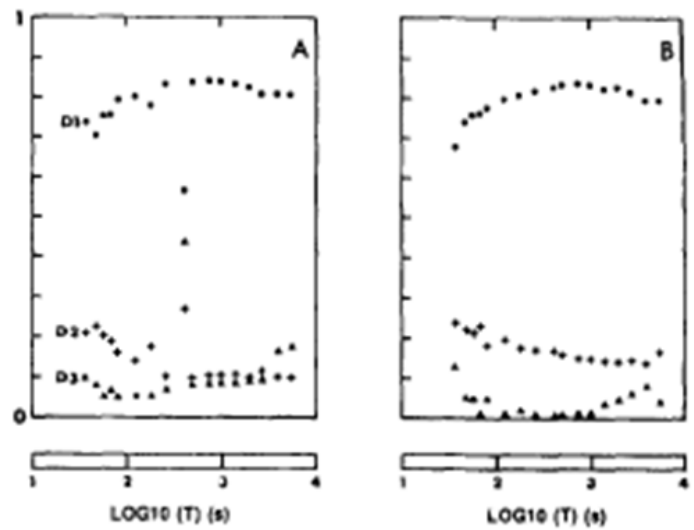

(a)
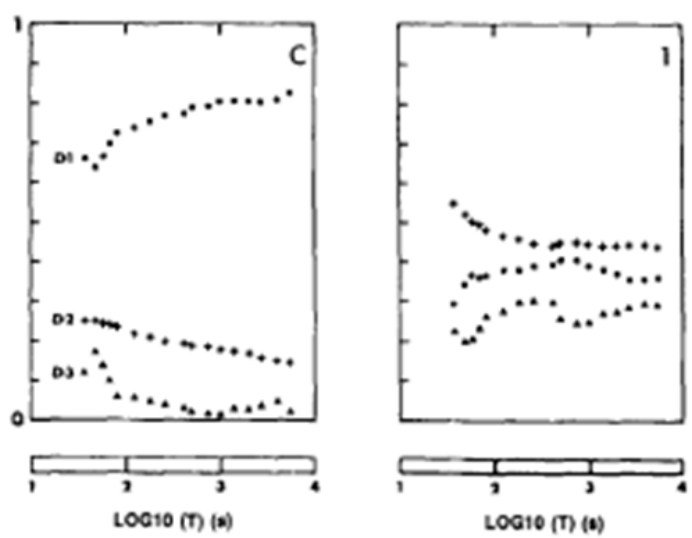

(b)
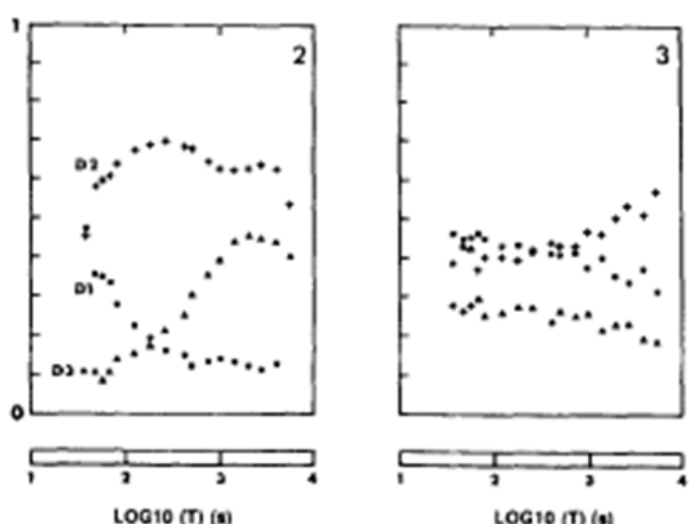

(c)

FIG. 4. The dimensional weights $D 1, D 2$, and $D 3$ for the six data sets. $D 1=$ solid circles. $D 2=$ vertical crosses. $D 3=$ solid triangles. 


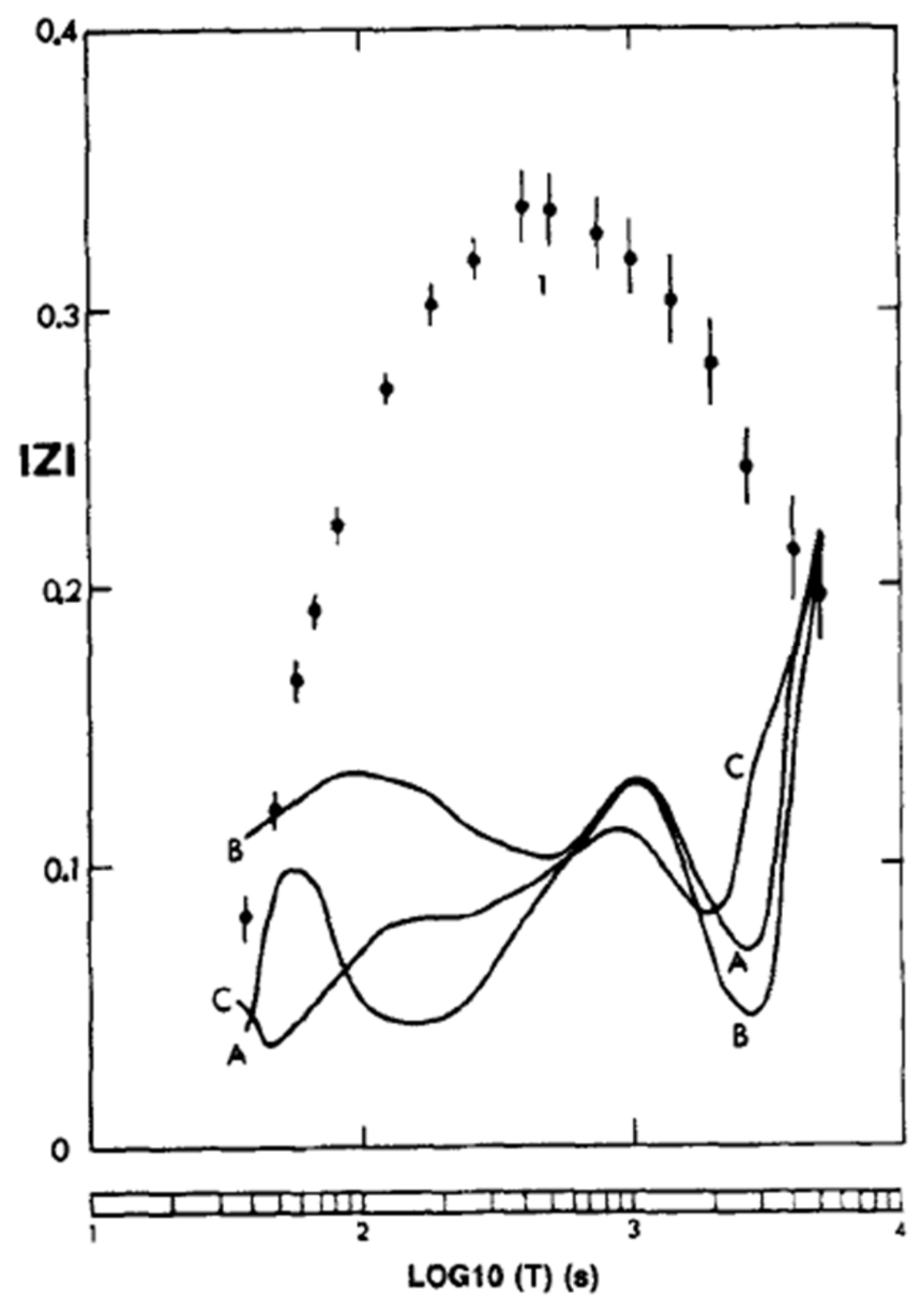

FIG. 5. The magnitude of the vertical field transfer function at the three central sites $(A, B$, and $\mathrm{C}=$ continuous curves) and at site 1 (discrete estimates). Typical 68 percent confidence limits are shown for the results at site 1. 


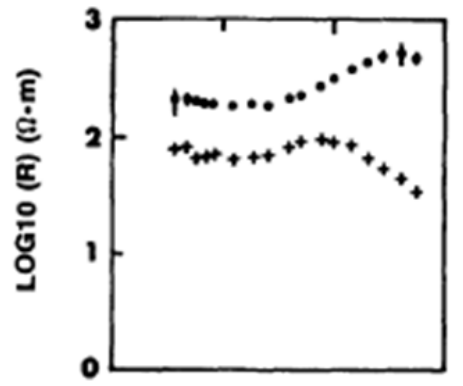

A
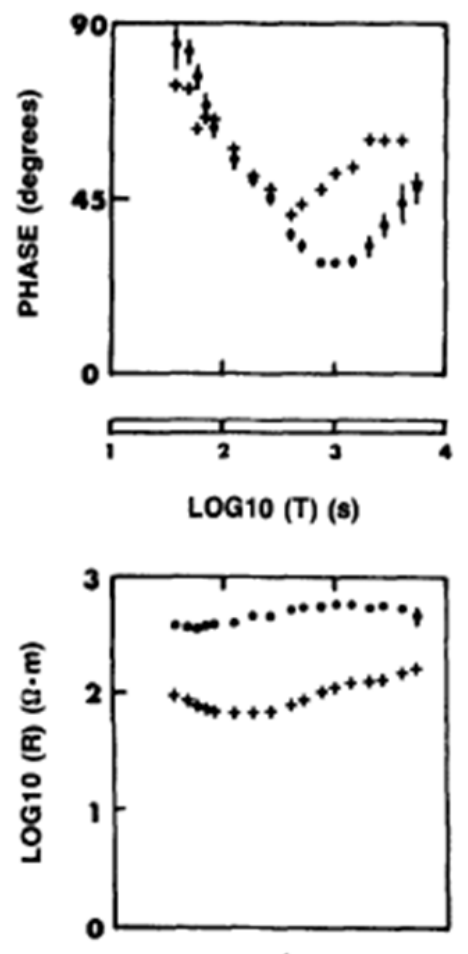

1

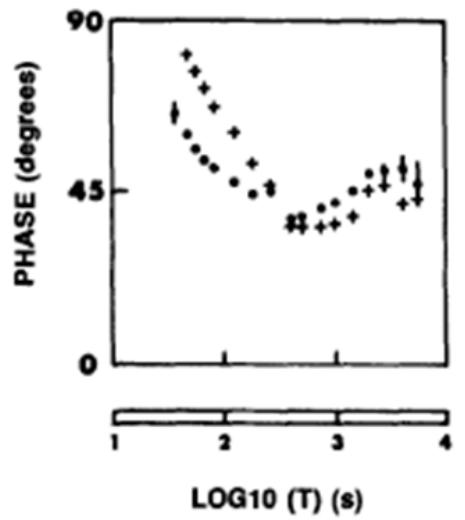

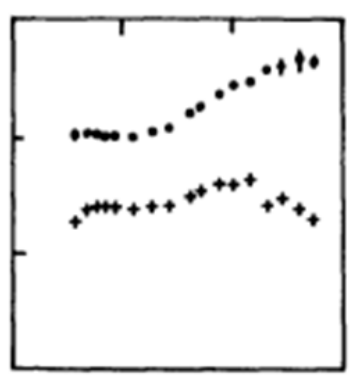

B
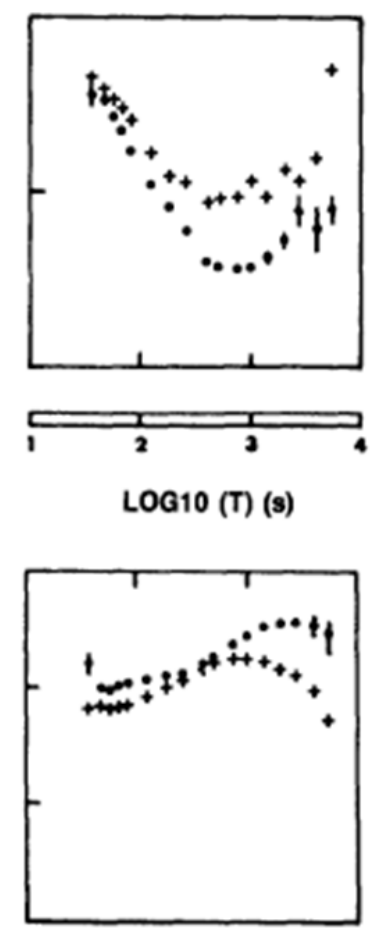

2

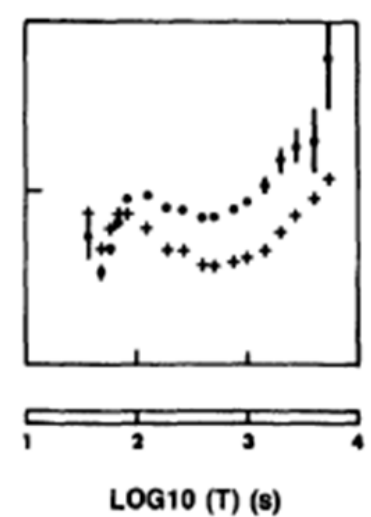

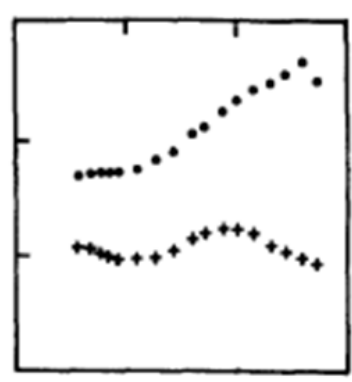

C
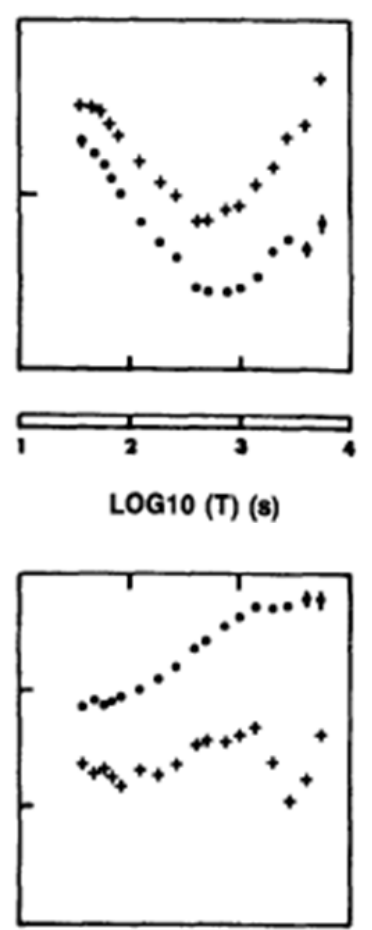

3

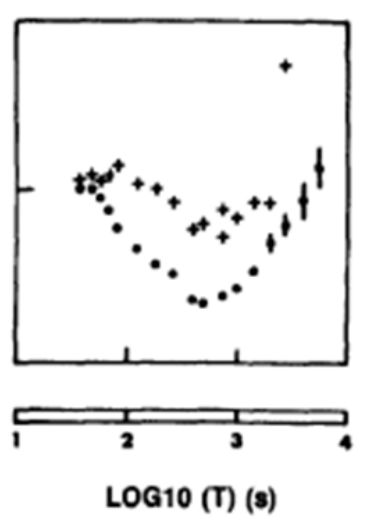

FIG. 6. Sounding curves for the six data sets rotated to principal directions. Apparent resistivity $(\mathrm{R})$ and phase in the major (solid circles) and minor (vertical crosses) directions at each site. Error bars correspond to 68 percent confidence limits. 


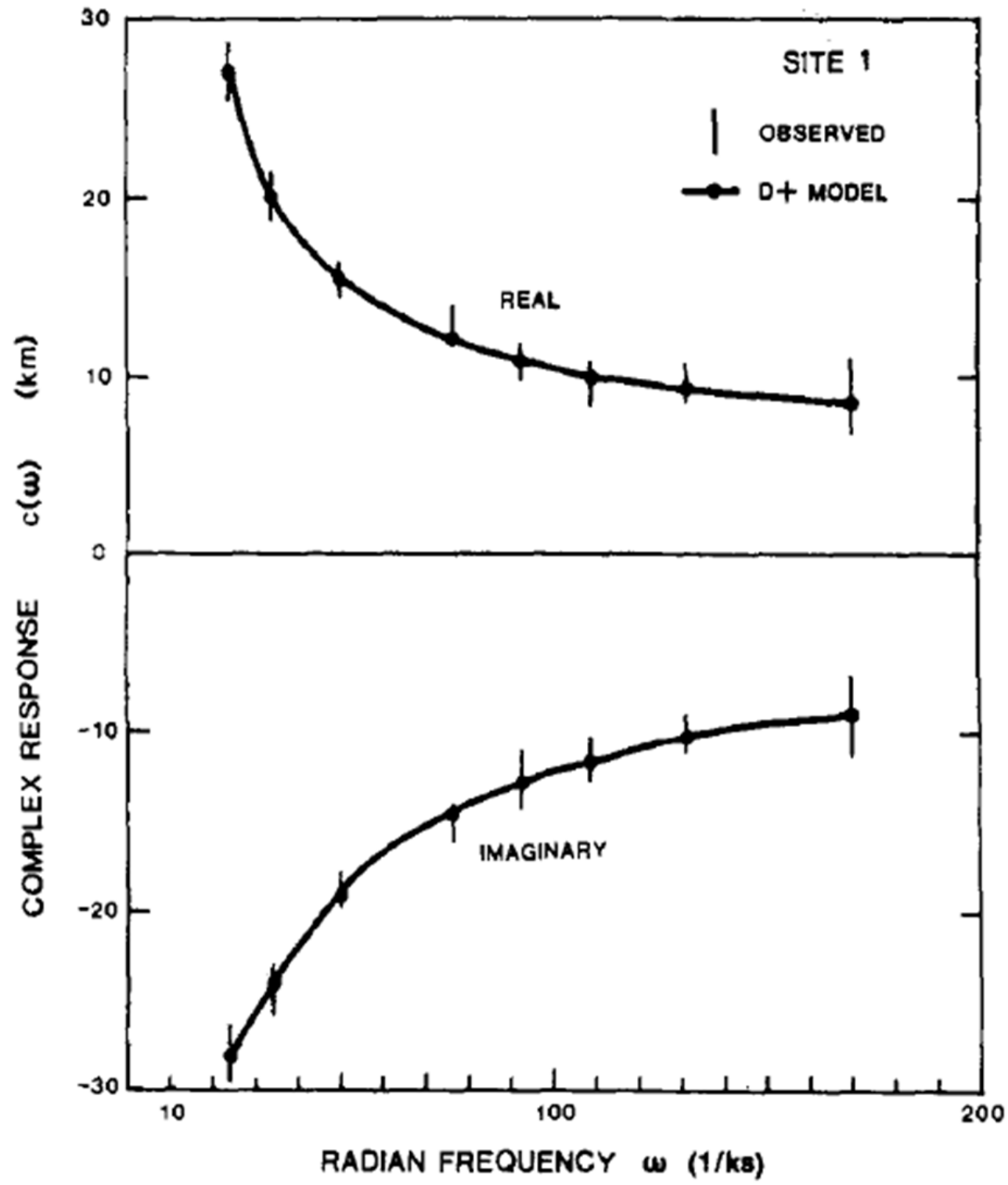

FIG. 7. Comparison of observed complex response (real and imaginary parts) at site 1 with that obtained from the $D+$ model in Table 2 . Vertical bars refer to observed values within two standard errors ( \pm one staridard error about the mean). 

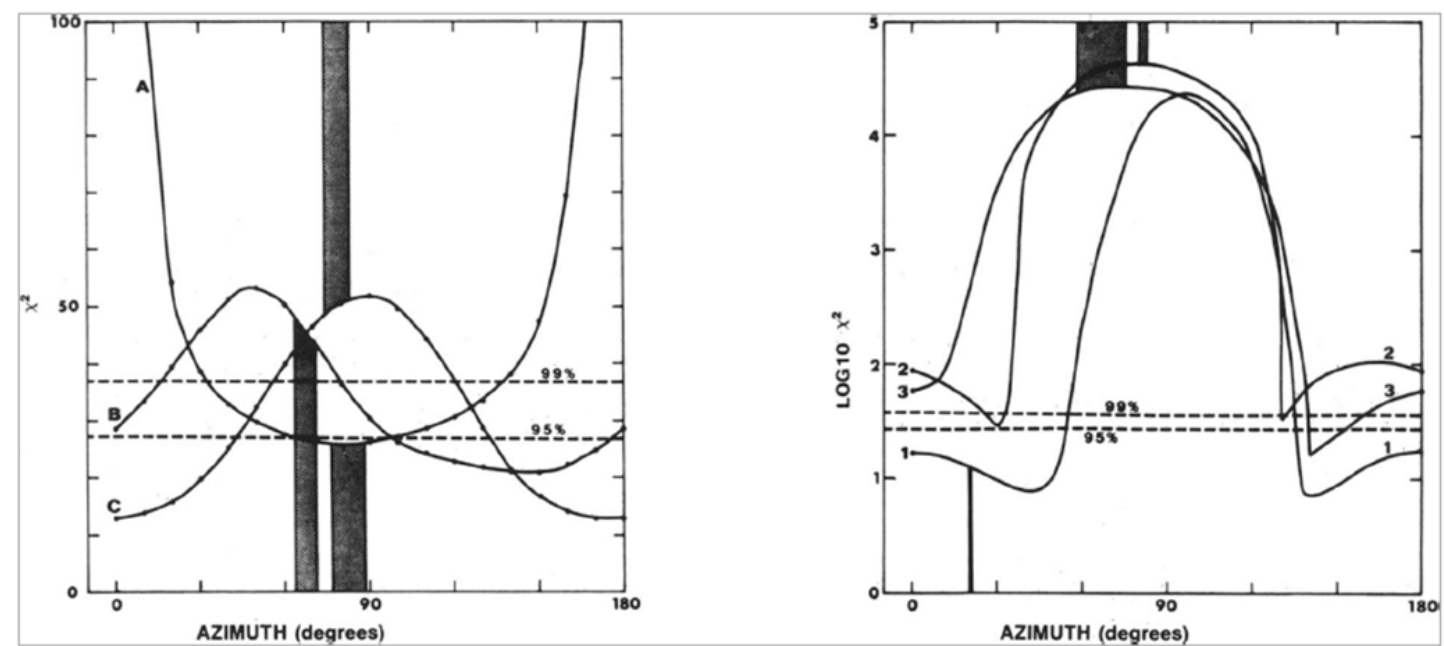

FIG. 8. Variation of the $\chi^{2}$ misfit of the optimum $D+$ solution (continuous lines) as a function of rotation azimuth in degrees east of magnetic north. The 95 percent and 99 percent $\chi^{2}$ acceptance limits are shown as broken horizontal lines. The data bandwidth is from 30 to $400 \mathrm{~s}$, and the azimuth range of the major principal axis for each data set is shown by the shaded region against the corresponding misfit curve for that site. (a) Linear scale for data from sites A, B, and C. (b) Logarithmic scale for data from sites I, 2, and 3. 